\title{
Software GeoGebra como herramienta en enseñanza y aprendizaje de la Geometría
}

\author{
Chiquinquirá Álvarez-Melgarejo * \\ Julieth Dayana Cordero - Torres"* \\ José Gilberto González Bareño **** \\ Omaida Sepúlveda-Delgado****
}

Artículo de investigación

Fecha de Recepción: 3 diciembre 2017

Fecha de Aprobación: 18 abril 2018

\section{Resumen}

En el artículo se presenta el uso de las TIC en el aula, como un recurso fundamental para el estudio: del desarrollo del pensamiento geométrico de los estudiantes. En el estudio realizado, se propuso como objetivo: diseñar e implementar sesiones didácticas de Geometría, haciendo uso del software GeoGebra en busca del fortalecimiento del pensamiento espacial de los estudiantes de grado cuarto y sexto. El grupo de estudio correspondió a estudiantes inmersos en una población rural con edades que oscilaron entre 9 y 12 años de edad de dos sedes rurales y la sede central de la Institución Educativa San Luis Beltrán del municipio de Covarachía. La Como metodología se trabajó la investigación acción. En el desarrollo de la fase de diseño, se tomaron como herramientas teóricas al enfoque Ontosemiótico del conocimiento y la instrucción matemática y la Teoría de las Situaciones Didácticas para la realización del análisis didáctico de las sesiones de clase. Se emplearon instrumentos como el diario de campo, la entrevista, los testimonios y el análisis a priori y a posteriori de la unidad didáctica, en cada clase, siguiendo los procesos de idoneidad didáctica, con el fin de analizar la importancia y el impacto que ocasiona en el contexto educativo, el uso de la tecnología como herramienta didáctica para la enseñanza y el aprendizaje de la geometría.
"Institución Educativa San Luis Beltrán - Boyacá Colombia

Chialme_2014@ootlook. com

** Institución Educativa San Luis Beltrán - Boyacá Colombia anayad_0329@hotmail.com *** Institución Educativa San Luis Beltrán - Boyacá Colombia

jg.Ing.Ambiental@gmail. com

****Universidad Pedagógica y Tecnológica de Colombia - Boyacá - Colombia Omaida.sepulveda@uptc. edu.co

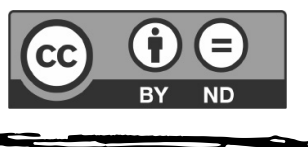


Entre los resultados, se pudo establecer que la estrategia didáctica correspondiente al diseño e implementación de un análisis didáctico a priori basado en los resultados arrojados en la etapa del diagnóstico: permitió tener una visión general de la clase de matemáticas y específicamente de los conocimientos previos de los estudiantes. A partir del diagnóstico y tomando como base los referentes de calidad, como: Los Lineamientos Curriculares, las Mallas Curriculares y los DBA: específicamente los procesos y competencias relacionados con el desarrollo del pensamiento espacial y los sistemas geométricos para el grado cuarto y sexto, se diseñaron e implementaron situaciones problema y secuencias didácticas que permiten fortalecer las competencias geométricas débiles, detectadas en la fase de diagnóstico. Se utilizó el programa Geogebra, como medio de interacción del estudiante para lograr el aprendizaje, según lo propuesto por la Teoría de las situaciones didácticas.

Palabras claves: Software GeoGebra, competencias, enfoque Ontosemiótico del conocimiento y la instrucción matemáticas, Teoría de las situaciones didácticas. 


\section{Introducción}

La evolución de la tecnología, trae al contexto educativo nuevas herramientas y con ello grandes cambios que implican romper paradigmas, hasta lograr en los educandos un aprendizaje significativo basado en una enseñanza para la comprensión, como lo plantean los estándares básicos de competencias en matemáticas. (EBC, 2006). La significatividad del aprendizaje no se reduce a un sentido personal de lo aprendido, sino que se extiende a su inserción en las prácticas sociales con sentido, utilidad y eficacia. La comprensión se entiende explícitamente como relacionada con los desempeños de comprensión, que son actuaciones, actividades, tareas y proyectos en los cuales se muestra la comprensión adquirida y se consolida y se profundiza la misma (MEN 2004). En las dimensiones de la comprensión se incluye no solo la más usual de los contenidos y sus redes conceptuales, sino que se proponen los aspectos relacionados con los métodos y técnicas, las formas de expresar y comunicar lo comprendido con la praxis cotidiana, profesional o científico-técnica en la que se despliega dicha comprensión según lo planteado en los estándares básicos de competencias en matemáticas. En esta dirección, se hace imprescindible implementar metodologías que generen habilidades y conocimientos y que lleven a desarrollar el pensamiento espacial, motivando al educando a mejorar su capacidad de análisis y de síntesis.

Realizar un proceso investigativo sobre el uso de herramientas tecnológicas para la realización de los procesos de enseñanza y aprendizaje en una institución educativa, produce en los estudiantes experiencias fascinantes y novedosas en el campo de la representación de los conceptos matemáticos, donde el uso de software les permite la utilización de algunas herramientas tecnológicas que llevan a facilitar el aprendizaje, rompiendo el paradigma que dificulta relacionar los conceptos geométricos con las vivencias personales. Por tal razón, para el estudio fue preciso hacer, en un primer momento un análisis de los estudios realizados en el orden internacional, nacional y local en el tema objeto de estudio y en especial, el análisis de los aportes que cada uno de ellos proporcionaba al trabajo investigativo.

En el contexto internacional Ruiz \& Atrio (2013), en su tesis: influencia del software de geometría dinámica GeoGebra en la formación inicial del profesorado de primaria, destaca que se usó el software para examinar la influencia de la herramienta en el proceso de enseñanza y aprendizaje de las matemáticas, para llegar a determinar si se mejoraban las competencias geométricas y didácticas de los estudiantes a cargo de un grupo de docentes. La investigación siguió una metodología cuasi-experimental y en los resultados se evidenció el mejoramiento en el desarrollo de procesos y habilidades geométricas relacionadas con el contexto y no aislados con respecto al lápiz y papel. Como conclusión se estableció que el software podía conducir al logro del desarrollo de las competencias
En el contexto internacional Ruiz \& Atrio (2013), en su tesis: influencia del software de geometría dinámica GeoGebra en la formación inicial del profesorado de primaria, destaca que se usó el software para examinar la influencia de la herramienta en el proceso de enseñanza y aprendizaje de las matemáticas 


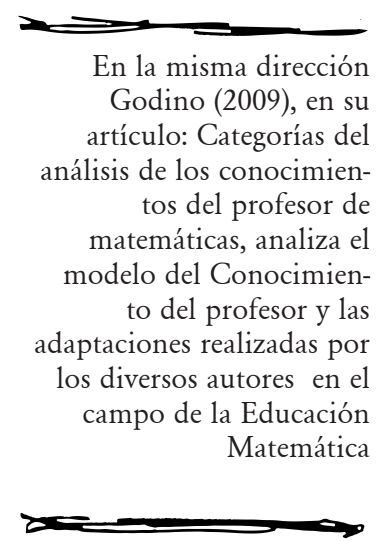

geométricas, sin tener en cuenta el nivel de conocimiento digital de los educandos.

En el contexto nacional una investigación quesoportala investigación encontramos a García (2011), en su trabajo de tesis doctoral "Evolución de Actitudes y Competencias Matemáticas en Estudiantes de Secundaria al Introducir Geogebra en el Aula", realiza una investigación que apunta al desarrollo $\mathrm{y}$ evolución tanto de las competencias matemáticas, en cuanto a la solución de problemas, donde se puede diseñar, poner en práctica y evaluar una secuencia de enseñanza basada en el uso de Geogebra que promueva una transformación positiva de las actitudes relacionadas con las matemáticas y un desarrollo de las competencias matemáticas de los estudiantes. Con una metodología denominada de investigación-acción y experimentos de enseñanza transformativos y dirigidos por una conjetura; proyecto que consistió: En la implementación de GEOGEBRA como herramienta para desarrollar actitudes y competencias matemáticas, en lo relacionado con geometría en estudiantes de secundaria. Una de las conclusiones obtenidas fue que se evidenció una transformación positiva de las actitudes relacionadas con las matemáticas de la mayoría de los estudiantes, gracias al trabajo con Geogebra. Además de generar resultados bastante satisfactorios, que muestran un desarrollo notable de determinadas competencias matemáticas.

Enlamismadirección, lasinvestigaciones de Argudo (2013), Castellanos (2010) y García (2011), resaltaron el hecho que la implementación del software GeoGebra para la enseñanza de la geometría, relacionada con el proceso de evaluación, generó un cambio en las actitudes y competencias matemáticas de los estudiantes. De las investigaciones, se concluye que se enfocaron a la reflexión de prácticas educativas, con una metodología basada en la investigación acción donde se plantearon talleres con el uso del software, los cuales arrojaron resultados positivos en cuanto a los procesos de enseñanza y aprendizaje relacionados con los procesos de evaluación.

En la misma dirección Godino (2009), en su artículo: Categorías del análisis de los conocimientos del profesor de matemáticas, analiza el modelo del Conocimiento del profesor y las adaptaciones realizadas por los diversos autores en el campo de la Educación Matemática en el tema: en este artículo se plantean unas categorías para el análisis de los conocimientos didácticos y matemáticos del docente según el enfoque Ontosemiótico del conocimiento y la instrucción matemática (EOS), donde se articulan las dimensiones epistémica (significados institucionales o socioculturales) y la cognitiva (significados personales, psicológicos o individuales) las cuales sirven como herramienta para abordar el análisis de los problemas de enseñanza y aprendizaje en el aula, modelando los propios conocimientos a enseñar y los aprendizajes logrados por los estudiantes. 
En la misma perspectiva, en las memorias del 11으 Encuentro Colombiano de matemática Educativa, Acosta (2010), en su artículo: Enseñando transformaciones geométricas con software de geometría dinámica, propuso como objetivo general: realizar una reflexión crítica del uso de la tecnología, las ventajas, los efectos y la transformación de la práctica educativa, en cuanto a la enseñanza de las matemáticas, basándose en la Teoría de las situaciones didácticas de Brousseau (1993), en cuanto a un aprendizaje por adaptación, con fundamentos y métodos de la didáctica de la matemática como son: la intención, el sujeto, la acción - retroacción- validación y un medio que para el caso correspondía a un software de geometría dinámica (SGD). El artículo sirvió como modelo, para analizar como la Teoría de las situaciones didácticas provee de herramientas teóricas para el análisis del aprendizaje, donde el que SGD se considera como un medio adecuado para la interacción de los alumnos, con la pretensión de que se produzca un aprendizaje.

Según los antecedentes analizados para el desarrollo de la investigación, se vio la necesidad de implementar una propuesta que relacionará la geometría plana que se trabaja en dos dimensiones, con la geometría dinámica que se puede trabajar en dos y tres dimensiones, direccionada a una mejor comprensión por los estudiantes de los conceptos geométricos. Se pretendía con la implementación de estas herramientas de aprendizaje, su contribución significativa para que los estudiantes llegaran a relacionar su aprendizaje con las vivencias del entorno, ya que el contexto educativo en el que se desarrolla el estudio, evidenció que la enseñanza de la geometría era un aspecto que se manejaba con clases magistrales, escasas herramientas tecnológicas y de un acceso a internet limitado, lo cual dificultaba y direccionaba el desarrollo del estudiante, minimizando su comprensión y evidenciando resultados desfavorables en el área. Según los argumentos presentados, surgió la necesidad de enseñar de un modo en que los estudiantes se involucraran con su entorno a través de herramientas didácticas.

Un factor que justificó el desarrollo de la investigación, fue el contexto del estudiante: una comunidad donde el 95\% de las personas habitan en la zona rural y son de escasos recursos como lo menciona el EOT (esquema de ordenamiento territorial) (2007) del Municipio de Covarachía. En la Institución Educativa San Luis Beltrán de este municipio, se observó que la educación en el área de matemáticas y específicamente en geometría no había tenido ningún tipo de evolución, ya que las dificultades en el aprendizaje de la geometría se continuaban evidenciando, esto según los resultados del análisis a las pruebas SABER $3^{\circ}, 5^{\circ}, 9^{\circ}, 11^{\circ}$ del año 2016: dificultades que fueron más notorias en las preguntas que valoraban los procesos de razonamiento geométrico y en la aplicación de conceptos para la solución de problemas. 
Un punto de reflexión importante, fue el hecho, que el software de geometría dinámica GeoGebra, se podía implementar en las zonas rurales donde los recursos didácticos eran escasos. Con base en la problemática planteada, el desarrollo de todo el proceso investigativo, el cual permitió analizar la importancia y el impacto que produce la utilización de la herramienta tecnológica GeoGebra en el contexto educativo, con la pretensión del logro de un aprendizaje significativo y aplicable a diferentes aspectos de la vida cotidiana del estudiante: para esto, se aplicó la metodología propuesta por el enfoque Ontosemiótico (Godino, 2011), según las categorías de análisis en cuanto a los indicadores de idoneidad didáctica para los procesos de enseñanza y aprendizaje de las matemáticas como se observa en la Figura 2.

Bajo estas perspectivas, los propósitos planteados en la investigación correspondieron a la realización de un análisis didáctico a priori para la clase de matemáticas, tomando como referencia el proceso de enseñanza y aprendizaje de la geometría, y la importancia del software GeoGebra en el fortalecimiento de las competencias matemáticas y geométricas, donde un factor importante fue la utilización del software como herramienta para el aprendizaje de la geometría la cual hace uso de la aplicación de las tecnologías en el aula.

La metodología empleada en el estudio se basó en la Investigación - Acción, según Elliot (2005), con la ayuda de instrumentos de recolección de información como el diario de campo, entrevistas, cuestionarios, grabaciones y la técnica de observación directa que parte de la misma práctica de los docentes investigadores y del análisis al contexto educativo, así como de las concepciones de los docentes acerca de la didáctica implementada para la enseñanza de la geometría; el estudio del currículo y del análisis a los resultados de las pruebas saber de los años 2015 y 2016, los cuales arrojaron un bajo porcentaje en las competencias matemáticas y en los procesos matemáticos como son: el razonamiento, comunicación $\mathrm{y}$ resolución de problemas, donde se destaca específicamente el componente del pensamiento espacial como lo muestra el Índice Sintético de Calidad Educativa de la Institución Educativa San Luis Beltrán (ISCE, 2016).

La investigación es de tipo mixto, donde se contemplan aspectos cualitativos y cuantitativos (Sampieri, 2014) ya que los dos enfoques permiten emplear procesos sistemáticos y empíricos para generar conocimiento: los datos cuantitativos son definidos y objetivos; se utiliza para el análisis de los datos, el enfoque Ontosemiótico y la Teorías de las situaciones didácticas: los datos cualitativos dan profundidvad, flexibilidad y permiten reconstruir la realidad del contexto escolar. El análisis de la práctica docente y el aprendizaje de los estudiantes se basan en observaciones directas, en la cuales los docentes investigadores informaban con objetividad, claridad y precisión las observaciones realizadas en el 
entorno de la Institución Educativa San Luis Beltrán, en el grado cuarto de la sede Chivascoque y el grado sexto de la sede central y de la Sede Antonio Nariño. En total, la población de estudio corresponde a 20 estudiantes cuyas edades oscilan entre 9 y 12 años: esta población se encuentra inmersa en una comunidad rural con un estrato socioeconómico uno; la población sujeto de investigación es tomada de las sedes educativas donde desarrollan la práctica educativa los docentes investigadores.

El estudio se considera de tipo exploratorio, ya que según Arias (1999): "La investigación exploratoria es aquella que se efectúa sobre un tema u objeto poco conocido o estudiado, por lo que sus resultados constituyen una visión aproximada de dicho objeto" (p. 46). Con el estudio se busca estudiar o explorar el desarrollo del pensamiento espacial en un contexto específico, utilizando una estrategia didáctica con la implementación de las TIC en el aula como medio o recurso; de igual forma se considera que el conocimiento se construye siempre por intereses que parten de las necesidades de los grupos, enfocándose en resolver los problemas encontrados en el contexto educativo inmerso en la investigación, como el bajo rendimiento académico, los resultados en pruebas saber, la apatía frente al aprendizaje de la geometría por parte de los estudiantes, la falta de contextualización de los conceptos básicos. Para dar solución a la problemática planteada, la investigación se apoya en la participación de todos los actores de la comunidad, en especial se tiene como información relevante para el desarrollo del proceso investigativo, el modelo educativo adoptado por la institución en el PEI (2015), que corresponde al modelo constructivista, donde se concibe la enseñanza como una actividad crítica y al docente, como un profesional autónomo que investiga y reflexiona sobre su práctica pedagógica, tomando la enseñanza no como la simple transmisión de conocimientos, sino como la organización pedagógica que permite al educando construir su propio saber.

Bajo estos supuestos, se espera del estudio, que pueda contribuir a un desarrollo efectivo de la Sociedad de la Información y del Conocimiento (SIC) dentro de la institución, ya que se debe asumir el reto de la alfabetización digital de los estudiantes, para que puedan hacer un uso libre y responsable de los recursos con o sin internet, interactuando con las TIC dentro del aula.

La investigación acción, según Elliot (2005), presenta varios ciclos divididos a su vez en diferentes fases: la fase de diagnóstico, planeación, acción, implementación y evaluación. Para el desarrollo de las fases de la investigación se implementaron las siguientes etapas: el desarrollo metodológico de la investigación partió de la aplicación de instrumentos de recolección de información en la fase diagnóstica como diarios de campo, cuestionarios a docentes dentro de las técnicas de observación y análisis de contenido; para la fase
La investigación exploratoria es aquella que se efectúa sobre un tema u objeto poco conocido o estudiado, por lo que sus resultados constituyen una visión aproximada de dicho objeto 
de diseño e implementación se diseña e implementa la estrategia didáctica, basada en el enfoque Ontosemiótico del conocimiento y la instrucción de la matemática acerca del análisis didáctico a priori y posteriori y la Teoría de las situaciones didácticas, las cuales se analizan según los indicadores de idoneidad didáctica para los procesos de enseñanza y aprendizaje de las matemáticas (epistémica, cognitiva, Afectiva, ecológica, interaccional y mediacional). Se realizó Se plantearon dos sesiones didácticas a partir de la teoría de la situaciones didácticas donde se le plantearon situaciones problema a los estudiantes basados en el contexto rural en el cual se desenvuelven diariamente, relacionando dichas situaciones que le permiten al estudiante validar- informar v evaluar a través del software GeoGebra; Como el contexto de la investigación es netamente rural con escasos recursos y acceso limitado a entornos informáticos y a las tecnologías de la información y la comunicación, se diseñó una cartilla para el estudiante como guía para el manejo del software GeoGebra; Para la evaluación de la investigación se realizó el análisis a posteriori según las categorías de análisis del EOS, análisis de datos cualitativos y cuantitativos según las categorías y subcategorías de análisis planteadas basadas en instrumentos de recolección como rejillas, sesiones didácticas, cartillas. A través de lo anterior se relaciona las fases de la investigación acción con el EOS y la TSD como lo muestra la figura 1.

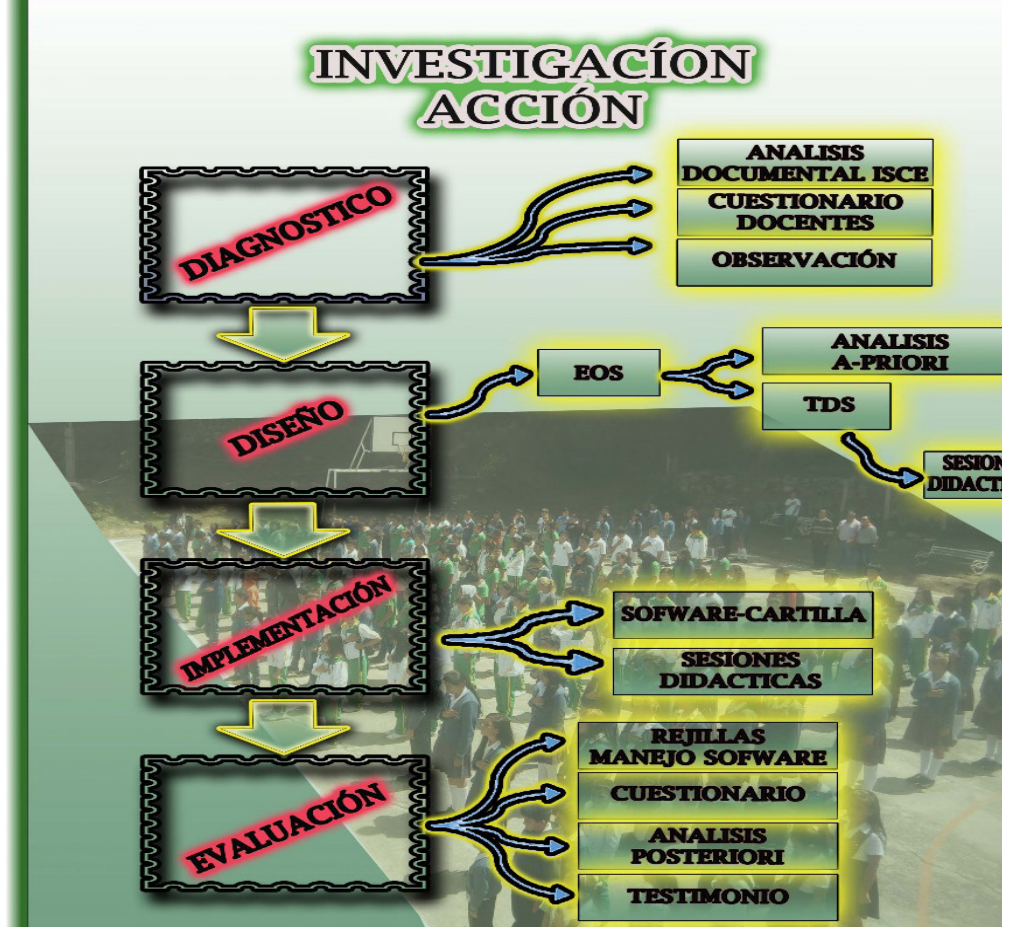

Figura 1: Interacción de fases de la investigación, el EOS y la TSD Fuente: autores de la investigación. 
Teniendo en cuenta los argumentos presentados, se planteó la pregunta de investigación: ¿Qué estrategia didáctica se puede diseñar e implementar en Geometría, para que los estudiantes de grado cuarto y sexto de la institución educativa San Luis Beltrán, haciendo uso del software GeoGebra, desarrollen las competencias geométricas? En esta dirección, se establecen como referentes teóricos al Enfoque Ontosemiótico del conocimiento y la instrucción Matemática (Godino, 2009) y La teoría de las situaciones didácticas (Brousseau 1978), los cuales sirvieron como marco para el planteamiento de un análisis didáctico a priori de la clase de matemáticas y el análisis a posteriori.-

\section{Referentes teóricos}

\section{Enfoque Ontosemiótico del conocimiento y la instrucción matemáticas-EOS}

El EOS, presenta unos indicadores de análisis que permiten evaluar la idoneidad didáctica de los procesos de enseñanza y aprendizaje de las matemáticas, según unos componentes e indicadores de calidad, los cuales proponen unas expectativas de adaptación, donde se toma la tecnología como un componente esencial del entorno (Godino, 2011); de igual forma, los indicadores permiten el análisis del rol del estudiante y del profesor frente a la enseñanza y el aprendizaje de las matemáticas, donde el estudiante de manera confiada se compromete con el desarrollo de tareas complejas, elegidas por los profesores; mientras que los profesores ayudan a los estudiantes a hacer y a explorar conjeturas sobre la base de la evidencia usando una variedad de razonamientos y técnicas de prueba para confirmar o rechazar las conjeturas. En la misma dirección, en Godino (2011), se plantean una serie de procesos que interactúan entre sí en la enseñanza y el aprendizaje de las matemáticas tales como: los afectos, el aprendizaje, el contenido, el contexto, los medios y las interacciones.

\section{COMPONENTES E INDICADORES DE CALIDAD}

\begin{tabular}{|c|c|c|}
\hline & $\begin{array}{l}\text { Se proponen unas } \\
\text { expectativas } \\
\text { ambiciosas para } \\
\text { todos, con adaptación } \\
\text { para aquellos que lo } \\
\text { necesitan. }\end{array}$ & $\begin{array}{l}\text { CONTENIDO } \\
\text { (Faceta epistémica) }\end{array}$ \\
\hline Figura 2. Indicadores de idoneidad & $\cdot \ldots$ & (Faceta interaccional) \\
\hline didáctica & $\begin{array}{l}\text { La tecnología es un } \\
\text { componente esencial } \\
\text { del entorno }\end{array}$ & \begin{tabular}{l|} 
MEDIOS \\
(Faceta mediacional)
\end{tabular} \\
\hline $\begin{array}{l}\text { Fuente: Indicadores de idoneidad } \\
\text { didáctica en procesos de enseñanza } \\
\text { y aprendizaje de las matemáticas } \\
\text { (Godino, 2011). }\end{array}$ & $\begin{array}{l}\text { Los estudiantes, de } \\
\text { manera confiada, se } \\
\text { comprometen con } \\
\text { tareas matemáticas } \\
\text { complejas elegidas } \\
\text { cuidadosamente por } \\
\text { los profesores. }\end{array}$ & $\begin{array}{l}\text { Los profesores ayudan a los estudiantes a hacer, } \\
\text { refinar y explorar conjeturas sobre la base de la } \\
\text { evidencia y usan una variedad de razonamientos y } \\
\text { técnicas de prueba para confirmar o rechazar las } \\
\text { conjeturas. }\end{array}$ \\
\hline
\end{tabular}


El EOS, representa un modelo apropiado para orientar y analizar los procesos de enseñanza y aprendizaje, porque desarrolla un sistema de nociones teóricas sobre la naturaleza, origen y significado de los objetos matemáticos desde una perspectiva educativa: este marco teórico, puede ser utilizado en otras áreas del conocimiento. El modelo teórico, plantea unas dimensiones de análisis de los procesos de estudio que nos permiten identificar y analizar los conocimientos matemáticos necesarios para el aprendizaje y la enseñanza de los conceptos matemáticos.

\section{DIMENSIONES DE ANÁLISIS DE UN PROCESO DE ESTUDIO}

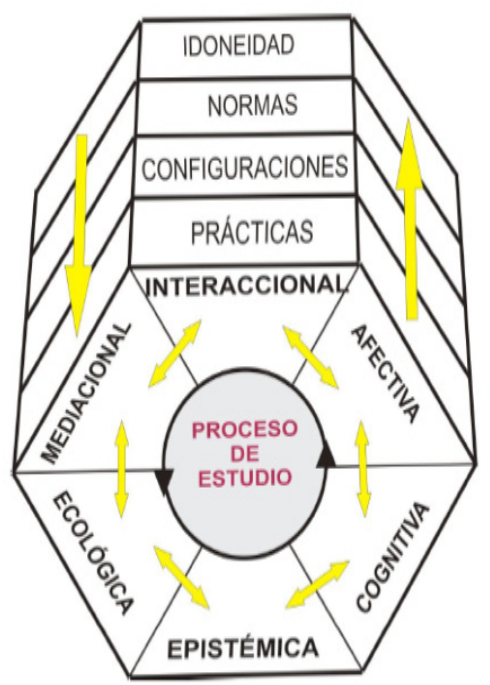

Figura 3. Facetas y niveles del conocimiento del profesor

Fuente: Indicadores de Idoneidad Didáctica de procesos de enseñanza y aprendizaje de las matemáticas Godino (2011).
Para analizar los procesos de instrucción matemática se propone el análisis de las siguientes facetas: a) Epistémica: referida a los conocimientos matemáticos pertinentes como estándares, malla curricular, DBA, contenidos; Cognitiva: relacionada con los conocimientos propios de los estudiantes sus pre-saberes, al igual que con las dificultades y avances que pueden presentar; Afectiva: relacionada con los intereses, las necesidades y actitudes de los estudiantes frente al aprendizaje que va a implementar, b)Ecológica: corresponde a la adaptación que se le hace al currículo teniendo en cuenta el aspecto social y la parte de la escuela y permite evaluar el uso del software en el contexto, c) Interaccional: se relaciona la interacción de los estudiantes y el profesor, para esta investigación la interacción del estudiante según la Teoría de las situaciones didácticas se realizó con el SGD, d) Mediacional: se relaciona con los recursos tecnológicos, la disponibilidad del tiempo, y los 
inconvenientes que se presenten con respecto a los recursos.

\section{Teoría de las situaciones didácticas}

La teoría de las situaciones didácticas propuesta por Brousseau (1978), plantea un aprendizaje por adaptación el cual es producto de la interacción del sujeto con el medio en que se vive sin la mediación del profesor. Según el autor, el aprendizaje biológico lo adapta al análisis de las actividades escolares a través de cinco fundamentos o métodos de la didáctica de la matemática, estos corresponde a: El sujeto parte de una intención respecto a una meta a alcanzar; realiza una acción sobre el medio; el medio reacciona a esa acción (retroacción); el sujeto interpreta la retroacción del medio usando los conocimientos de los que ya dispone y el sujeto valida su acción de acuerdo con la interpretación que hace de las retroacciones del medio.

Según estos argumentos, la Teoría de las situaciones didácticas, se basa en unas situaciones didácticas y situaciones a-didácticas; la primera referida a situaciones cotidianas de clase, y la segunda a la actividad que produce un aprendizaje por adaptación. En estos casos juega un papel importante la relación directa entre el saber (a enseñar), el profesor (que desea enseñar ese saber) y el alumno (que desean aprender ese saber).

Resultados: Desarrollo de la experiencia

La estrategia didáctica propuesta, correspondióaldiseñoeimplementación de un análisis didáctico a priori basado en los resultados arrojados en la etapa del diagnóstico, según las etapas propuestas en la Investigación Acción (Elliot, 2005). En esta etapa se logró obtener una visión general de la clase de geometría. A partir del diagnóstico y tomando como base los referentes de calidad: Lineamientos Curriculares, las Mallas Curriculares y los DBA, específicamente los procesos y competencias a trabajar en el pensamiento espacial y los sistemas geométricos para el grado cuarto y sexto, se pasó a la fase de acción, donde se diseñaron e implementaron situaciones problema y sesiones didácticas que buscaban fortalecer las competencias matemáticas. En la misma dirección, para que el estudiante conociera e interactuara con el software se elaboró una guía acerca del manejo del software como base para la implementación de las sesiones didácticas contextualizadas, con el objetivo de generar unos aprendizajes por adaptación, en concordancia con la Teoría de las situaciones didácticas. En la misma fase, se realizó un análisis didáctico a Priori, para organizar el proceso de instrucción y luego de esta planeación se pasa a su implementación y para analizar lo pretendido se realiza el análisis a posteriori, según lo plantea el EOS, para evaluar los resultados obtenidos y compararlos con los resultados del primer análisis y de esta manera realizar la retroalimentación de cada sesión implementada.

Los instrumentos utilizados para la recolección de datos e información y la adquisición de evidencias que
La teoría de las situaciones didácticas propuesta por Brousseau (1978), plantea un aprendizaje por adaptación el cual es producto de la interacción del sujeto con el medio en que se vive $\sin$ la mediación del profesor. 


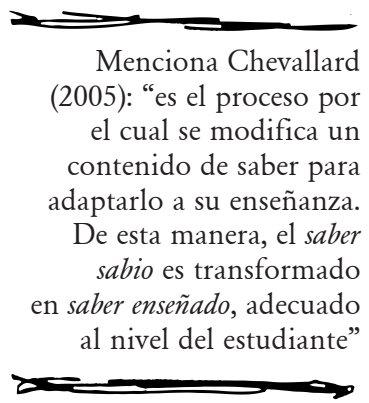

soportan la investigación se basaron en: diarios de campo de las clases observadas de los docentes del área de matemáticas, en las clases específicas de geometría; cuestionarios a docentes con entrevistas semi-estructuradas para identificar las estrategias de enseñanza, algunas concepciones de los docentes sobre la implementación de las TIC en el aula, análisis de la influencia de estas herramientas para el aprendizaje de los estudiantes, análisis documental de resultados de pruebas saber en los grados $3^{\circ}$ y $5^{\circ}$ de los años 2015 y 2016 : dibujos elaborados por los estudiantes para identificar la concepción que tenían sobre los avances de la enseñanza de la geometría en el transcurso de los años, fotografías, rejillas de evaluación, testimonios acerca de la concepción de los estudiantes en el desarrollo de la propuesta.

El análisis de los resultados, soportados en los instrumentos aplicados reflejaron los problemas de los educandos en el área de las matemáticas, específicamente con el pensamiento espacial y los sistemas geométricos. Según el análisis de los resultados en las pruebas externas: pruebas SABER de $3^{\circ}$ y $5^{\circ}$ de los años 2015 y 2016. El análisis de los resultados del cuestionario realizado a docentes en cuanto a sus metodologías, prácticas educativas y pedagógicas, reflejaron que el docente era consciente de que su práctica educativa, se basa en algunos de los métodos de la enseñanza tradicional. Adicionalmente, se evidenciaron algunas falencias en el currículo en cuanto a la enseñanza de la geometría, ya que el tiempo era limitado y superficial, según lo muestran los planes de aula y de área en la Institución Educativa. De igual forma, la información reveló que los educandos percibían falencias en el proceso de enseñanza y aprendizaje y mostraron en gráficos el interés por explorar nuevas situaciones, viendo viable la implementación de nuevas estrategias didáctica.

Teniendo en cuenta el diagnóstico realizado y en busca de una solución a la problemática se planteó a la comunidad educativa la utilización del software en geometría dinámica. Inicialmente se dio a conocer el software GeoGebra a los docentes a través de un taller participativo, en la búsqueda de nuevas alternativas que permitieran mejorar el que-hacer docente y que condujeran a una transformación de las estrategias pedagógicas, como lo menciona Chevallard (2005): "es el proceso por el cual se modifica un contenido de saber para adaptarlo a su enseñanza. De esta manera, el saber sabio es transformado en saber enseñado, adecuado al nivel del estudiante" (p. 45).

Los resultados de la implementación de la cartilla como inducción para el manejo del software GeoGebra, fue positivo, los estudiantes lograron manejar las diferentes herramientas del SGD, desarrollando las guías de trabajo que le permitió relacionar lo dibujado en lápiz y papel y lo representado en GeoGebra; para el desarrollo de las sesiones didácticas, resultado del análisis didáctico, para ser aplicadas. Los estudiantes identificaron la situación problema con facilidad ya que se les presentó de 
manera contextualizadas como aplicar conceptos básicos de geometría y aspectos para potenciar el pensamiento espacial, trabajados con base en los cultivos que las familias de los mismos estudiantes, donde se evidenció cierto grado de dificultad al relacionar las tres visiones, para aplicar acciones en la situación problema, que correspondió a asignar cultivos a diferentes terrenos graficándolos en las guías de trabajo de las sesiones y su representación y solución en GeoGebra. Como se evidencia las imágenes a continuación:
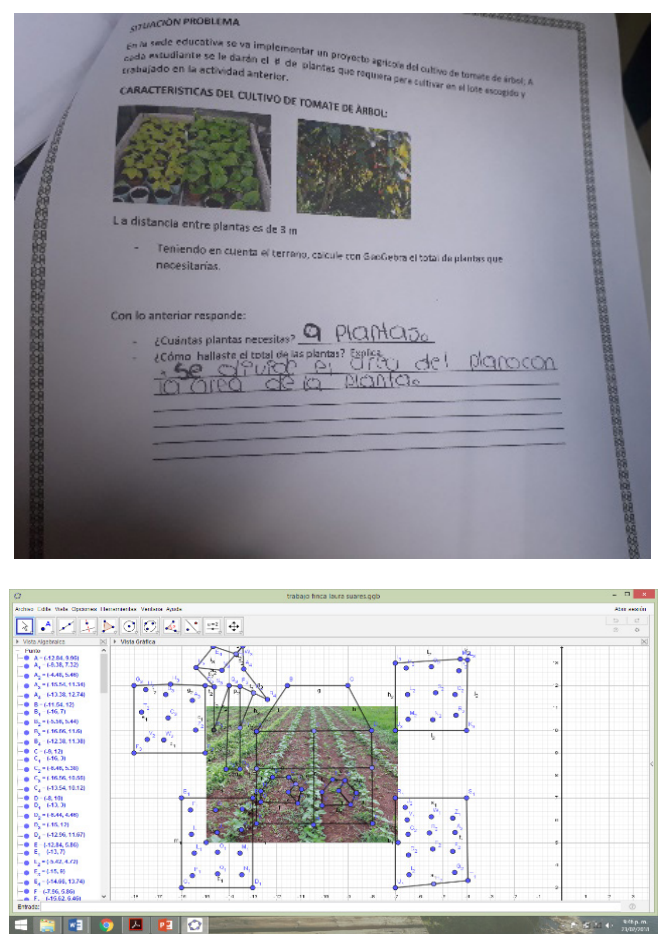

Evidencias fotográficas de trabajos realizados

En el análisis a posteriori se evidenció que se lograron los objetivos planteados en cada sesión según las facetas propuestas para el análisis de la idoneidad didáctica: se observó la interacción activa de los estudiantes con el software GeoGebra y con el contexto según el planteamiento y análisis realizado a la faceta interaccional. Se lograron aclarar dudas acerca del manejo del software y de conceptos básicos de geometría como parte del análisis efectuado en la faceta cognitiva; de igual forma, los estudiantes lograron un aprendizaje por adaptación de acuerdo a la teoría de las situaciones didácticas y a-didácticas, potenciando el pensamiento espacial con base en estándares básicos de competencias dentro del estudio de la faceta epistémica. Se realizó una adaptación de sesiones y situaciones didácticas de acuerdo a intereses y características de los estudiantes que sirvieron como población de estudio dentro del desarrollo de la investigación como parte del análisis y estructuración de la faceta ecológica; también, se pudo evidenciar el interés y la motivación en los estudiantes por aprender cada vez más por el uso del Software, porque estaban familiarizados con las situaciones planteadas como pretensión en el planteamiento de la faceta afectiva; y finalmente, se lograron solucionar algunos inconvenientes que se iban presentando desde un inicio con la idea de llevar los recursos informáticos y las TIC a este contexto escolar, con oportunidades mínimas de acceso a dichas tecnologías.

\section{Consideraciones Finales}

Un primer problema que se aborda, corresponde al análisis del contexto 
inmerso en la experiencia, ya que se debía partir de los pre-saberes de los educandos: no se puede dejar de lado la realidad en la que se encuentran los estudiantes. Es por esto que al considerar su contexto como la base fundamental del proceso de enseñanza y aprendizaje, los resultados de aprendizaje llegaron a ser más significativos, ya que, aun estando inmersos en un contexto con escasos medios de comunicación y de información, el impacto de la herramienta tecnológica fue innovador.

De igual manera se observó que el uso de las TIC en el aula, se relaciona con aprendizajes previos de los estudiantes, con situaciones cotidianas y actividades extracurriculares; de esta manera al llevar situaciones propias del contexto con el software GeoGebra se evidenció un aprendizaje más significativo.

Otro aspecto a resaltar, es el hecho que el uso del software de geometría dinámica, en este caso el GeoGebra, tiene un impacto relevante al ser implementada en al aula de clase ya que permite el fortalecimiento de las competencias geométricas y lleva a los estudiantes a plantear una conjetura final para poder pasar a verificarla.

En cuanto al análisis didáctico de las sesiones de clase, se pueden considerar como un instrumento a través del cual el profesor, organiza y programa los procesos de enseñanza y aprendizaje que van a desarrollar con sus estudiantes, esto facilita la orientación y la organización sistemática de los contenidos de las diferentes áreas del conocimiento alrededor de un tema, para satisfacer el trabajo en el aula; además por su carácter flexible permite la incorporación de recursos como apoyo y complemento, para ampliar las posibilidades de aprendizaje en los estudiantes y el enriquecimiento del acto comunicativo en el aula.

En la misma dirección, las TIC crean nuevas formas de interacción entre los actores del proceso formativo que favorecen la comunicación y los niveles departicipación:la aplicación delas TIC, permite que los estudiantes trabajen de manera activa y organizada actuando con autonomía en el desarrollo de sus tareas, estando atentos a las necesidades de cada uno de sus compañeros. Por su parte el rol del docente cambia notoriamente, pasa de ser un trasmisor de conocimientos a un facilitador y orientador, pues es él quien organiza la estrategia, estructuran los temas y diseñan las actividades pertinentes para enriquecer y evaluar el aprendizaje: $\mathrm{da}$ las explicaciones necesarias para el desarrollo de las temáticas y crea espacios para que los estudiantes utilicen los recursos informáticos, no solo como herramienta para enriquecer sus conocimientos, sino que además favorece el desarrollo de habilidades. En este sentido, el maestro es el mediador entre los conocimientos que ofrece la tecnología y sus estudiantes, un planificador y un diseñador de las actividades para utilizar con sentido pedagógico las Tecnologías de la información y la comunicación. 


\section{Referencias bibliográficas}

Acosta, M. (2010). Enseñando transformaciones geométricas con software de geometría dinámica. Escuela de matemáticas del grupo Edumat. Bucaramanga: Universidad Industrial de Santander.

Arias, F. (1999). El Proyecto de Investigación: Guía Para su Elaboración. Caracas: Episteme.

Argudo, M. (2013). Las Tic y el aprendizaje dela geometría. Tesis de Maestría. España: Universidad Cardenal Herrera: CEU.

Brousseau G. (1978). Observation desactivities didactiques. La Revue francaise de pedagogie $n^{-} 45$.

Brousseau G. (1993). Fundamentos y métodos de la Didáctica de la Matemática. Serie B. Trabajos de Matemática. No. 19. Facultad de Matemática Astronomía y Física. Versión Castellana. Universidad Nacional de Córdoba.

Castellanos, I. (2010). Visualización y razonamiento en las construcciones geométricas utilizando el software GeoGebra con alumnos de II de magisterio en la ENMP (Tesis de maestría). Honduras, Universidad Pedagógica Nacional Francisco Morazán: Tegucigalpa.

Chevallard, Y. (2005). La trasposición didáctica: del saber sabio al saber enseñado. Digitalizado por I.S.C. Héctor Alberto Turrubiartes Cerino.

Elliot (2005). El cambio educativo desde la investigación (4르. Edición): Editorial Morata.

EOT (2007). Esquema de ordenamiento territorial. Municipio de Covarachia: Boyacá (2007).

García, M. (2011). Evolución de actitudes y competencias matemáticas en estudiantes de secundaria al introducir el GeoGebra en el aula (Tesis doctoral). Almería, España: Universidad de Almería.

Godino, J. (2009). Categorías de análisis de los conocimientos del profesor de matemáticas. UNIÓN. Revista Iberoamericana de Educación Matemática, (20), 1331.

Godino, J. (2011). Indicadores de Idoneidad didáctica en procesos de enseñanza y aprendizaje de las matemáticas. http://www.urg.es/local/jgodino

ISCE (2016). Índice sintético de calidad educativa. http://aprende.colombiaaprende. edu.co/es/siemprediae I.E. San Luis Beltrán. Covarachía: Boyacá. 
E.B.C. (2006). Estándares Básicos de Competencias. En Lenguaje, Matemáticas, Ciencias y Competencias Ciudadanas (pág. 61). Bogotá: Imprenta Nacional de Colombia.

PEI (2015). Proyecto educativo Intitucional. Institución Educativa San Luis Beltrán. Covarachía: Boyacá.

Ruiz, N. \& Atrio, S. (2013). Influence of digital proficiency in geometric skills acquisition in GeoGebra. Madrid: Universidad Autónoma de Madrid.

Sampieri, R (2014). Metodología de investigación. (6 edición). México: Mc Graw Hill. 